\title{
Characterization of magnetic field noise in the ARIADNE source mass rotor
}

\author{
Nancy Aggarwal,,${ }^{1,2, *}$ A. Schnabel, ${ }^{3}$ J. Voigt, ${ }^{3, \dagger}$ Alex Brown, ${ }^{4,}$ J. C. Long, ${ }^{4}$ S. Knappe-Grueneberg, ${ }^{3}$ W. Kilian, ${ }^{3}$ A. Fang, ${ }^{5}$
} A. A. Geraci, ${ }^{1}$ A. Kapitulnik, ${ }^{5}$ D. Kim, ${ }^{6,7}$ Y. Kim, ${ }^{6,7}$ I. Lee, ${ }^{4}$ Y. H. Lee, ${ }^{8}$ C. Y. Liu, ${ }^{4}$ C. Lohmeyer, ${ }^{1}$ A. Reid, ${ }^{4}$ Y. Semertzidis, ${ }^{6,7}$ Y. Shin, ${ }^{6}$ J. Shortino, ${ }^{4}$ E. Smith, ${ }^{4}$ W. M. Snow, ${ }^{4}$ E. Weisman, ${ }^{1}$ and H. Zhang ${ }^{1}$

(ARIADNE Collaboration)

${ }^{1}$ Center for Fundamental Physics, Department of Physics and Astronomy, Northwestern University, Evanston, Illinois 60208, USA

${ }^{2}$ Center for Interdisciplinary and Exploratory Research in Astrophysics (CIERA), Northwestern University, Evanston, Illinois 60201, USA

${ }^{3}$ Physikalisch-Technische Bundesanstalt (PTB), Abbestraße 2-12, 10587 Berlin, Germany

${ }^{4}$ Department of Physics, Indiana University, 727 East Third Street, Bloomington, Indiana 47405, USA

${ }^{5}$ Department of Physics and Applied Physics, Stanford University, 382 Via Pueblo, Stanford, California 94305, USA

${ }^{6}$ Center for Axion and Precision Physics Research, IBS, 193 Munji-ro, Daejeon 34051, Republic of Korea

${ }^{7}$ Department of Physics, KAIST, 291 Daehak-ro, Daejeon 34141, Republic of Korea

${ }^{8}$ KRISS, 267 Gajeong-ro, Yuseong-gu, Daejeon 34113, Republic of Korea

(Received 2 December 2020; accepted 13 January 2022; published 3 February 2022)

\begin{abstract}
The Axion Resonant Interaction Detection Experiment (ARIADNE) is a nuclear-magnetic-resonance-based experiment that will search for novel axion-induced spin-dependent interactions between an unpolarized source mass rotor and spin-polarized ${ }^{3} \mathrm{He}$ nuclei placed nearby. To detect a feeble axion-mediated signal at the subattotesla level, the experiment relies on ultralow magnetic background and noise. We measure and characterize the magnetic field from a prototype tungsten rotor. We show that the field is dominantly caused by a few discrete magnetic dipoles, likely due to impurities in the rotor. This is done via a numerical optimization pipeline which fits for the locations and magnetic moments of each dipole. We find that under the current demagnetization procedure, the magnetic moment of the impurities is bounded at $10^{-9} \mathrm{~A} \mathrm{~m}^{2}$. We further show that a shielding factor of $10^{9}$ will support ARIADNE's design sensitivity with the current level of tungsten purity and demagnetization process.
\end{abstract}

DOI: 10.1103/PhysRevResearch.4.013090

\section{INTRODUCTION}

The QCD axion is a particle predicted to exist in order to explain the strong- $C P$ problem, connected with the lack of $C P$ violation observed in the strong interactions [1-4]. Experimental searches for a neutron electric dipole moment constrain the angle $\theta_{\mathrm{QCD}}$-which would be expected to be of order $1 \mathrm{rad}$ - to be less than $10^{-10} \mathrm{rad}[5,6]$. The axion provides a dynamical mechanism to explain the unnatural smallness of this angle. In addition, the axion is an excellent candidate to explain the dark matter in our universe.

The axion can mediate novel short-range spin-dependent forces between fermions. The Axion Resonant Interaction Detection Experiment (ARIADNE) will search for a spindependent interaction that is mediated by the QCD axion between a gas of hyperpolarized ${ }^{3} \mathrm{He}$ nuclear spins and an

\footnotetext{
*nancy.aggarwal@northwestern.edu

$\dagger$ Jens.Voigt@ptb.de

${ }^{\ddagger}$ Deceased.
}

Published by the American Physical Society under the terms of the Creative Commons Attribution 4.0 International license. Further distribution of this work must maintain attribution to the author(s) and the published article's title, journal citation, and DOI. unpolarized source mass [7,8] (refer to Fig. 1). In particular, the axion can mediate an interaction between nucleons with a potential given by [4]

$$
V_{s p}(r)=\frac{\hbar^{2} g_{s}^{N} g_{p}^{N}}{8 \pi m_{N}}\left(\frac{1}{r \lambda_{a}}+\frac{1}{r^{2}}\right) e^{-\frac{r}{\lambda a}}(\vec{\sigma} \cdot \hat{\vec{r}}),
$$

where $m_{N}$ is the unpolarized nucleon's mass, $\vec{\sigma}$ is the Pauli spin matrix, $\vec{r}$ is the vector between them, and $\lambda_{a}=\hbar /\left(m_{a} c\right)$ is the reduced Compton wavelength of the axion. For the QCD axion, the coupling constants $g_{s}^{N}$ and $g_{p}^{N}$ are related to the axion mass as they are fixed by the axion decay constant $f_{a}$ :

$$
6 \times 10^{-27}\left(\frac{10^{9} \mathrm{GeV}}{f_{a}}\right)<g_{s}^{N}<10^{-21}\left(\frac{10^{9} \mathrm{GeV}}{f_{a}}\right)
$$

and

$$
g_{p}^{N}=\frac{C_{f} m_{N}}{f_{a}}=C_{f} \times 10^{-9}\left(\frac{m_{N}}{1 \mathrm{GeV}}\right)\left(\frac{10^{9} \mathrm{GeV}}{f_{a}}\right),
$$

where $C_{f}$ is a dimensionless coupling constant of order 1 [7]. $g_{s}^{N} \propto \theta_{\mathrm{QCD}}$, where $\theta_{\mathrm{QCD}}$ is the $C P$-violating phase appearing in the Lagrangian describing the strong interactions.

Since the axion couples to $\vec{\sigma}$, which is proportional to the nuclear magnetic moment, the axion coupling can be treated as an effective magnetic field. The expected magnitude of this fictitious magnetic field depends on the scalar coupling 
$g_{s}^{N}$ and dipole coupling $g_{p}^{N}$ of the axion to nucleons. ARIADNE utilizes a nuclear-magnetic-resonance-based scheme in order to detect this spin-dependent interaction. The hyperpolarized spins, subject to an applied bias field $\left(\vec{B}_{\text {ext }}\right)$, will precess at the nuclear Larmor frequency set by the bias field.

If the effective axion field is perpendicular to $\vec{B}_{\text {ext }}$, it can resonantly drive the nuclear spin magnetization when modulated on resonance at the nuclear Larmor frequency, resulting in a transverse magnetization of the ${ }^{3} \mathrm{He}$ spins. This precessing transverse magnetization can then be measured using a superconducting quantum interference device (SQUID). The ${ }^{3} \mathrm{He}$ gas will be enclosed in an oblate ellipsoid with axis lengths $3 \mathrm{~mm}, 3 \mathrm{~mm}$, and $150 \mu \mathrm{m}$. To probe the heaviest possible axion mass in the experiment, this ellipsoid will need to be placed closest to the rotor, with the center of the ellipsoid being $200 \mu \mathrm{m}$ away from the rotor surface. Integrating over the geometry used in ARIADNE, the effective magnetic field due to the axion is expected to be of order $10^{-19} \mathrm{~T}$, or less, depending on the value of $g_{s}^{N}$. In order to distinguish the axion signal, real magnetic fields need to be shielded to a level below the expected fictitious magnetic field. In ARIADNE, a superconducting niobium magnetic shield will enclose the ${ }^{3} \mathrm{He}$ sample, separating the ${ }^{3} \mathrm{He}$ sample from the source mass and shielding the ${ }^{3} \mathrm{He}$ gas from ordinary electromagnetic field perturbations.

In the experiment, the modulation of the unpolarized mass, and subsequently the modulation of the axion field, will be implemented by rotating a tungsten sprocket that has 11 cutouts around its circumference. The modulation frequency $\left(f_{\text {mod }}=\right.$ $55-99 \mathrm{~Hz})$ will then be 11 times the rotation frequency $\left(f_{\text {rot }}=\right.$ 5-9 Hz). The subattotesla axion-induced effective magnetic field created by this mass is at $f_{\text {mod }}$ and is not shielded by the superconductor. The magnetic background from the rotor and the shielding factor of the superconducting shield together play a combined role in achieving the target axion sensitivity. For instance, assuming a baseline shielding factor of $10^{8}$, any coherent magnetic field background at the modulation frequency must be lower than $1 \mathrm{pT}$ in order for ARIADNE to operate at a sensitivity of $10^{-20} \mathrm{~T}$. Additionally, assuming an integration time of $10^{6} \mathrm{~s}$ and the same shielding factor, the magnetic field noise from stochastic processes (e.g., Johnson noise) must be lower than $1 \mathrm{nT} / \sqrt{\mathrm{Hz}}$. From this point on, we will refer to something as noise if its effect can be lowered by integrating for longer times, and as background if it does not get suppressed by integrating longer. Recent experimental testing has shown that using a combination of thin-film $\mathrm{Nb}$ sputtered on quartz and lead foil shielding, shielding factors of approximately $10^{8}$ should be realistic [9]. The ARIADNE cryostat and experiment are in the construction phase along with the testing of various components, including a low-noise SQUID gradiometer system, a ${ }^{3} \mathrm{He}$ nuclear polarization system, and a coil geometry to provide the appropriate magnetic environment for the ${ }^{3} \mathrm{He}$ sample.

Several relevant sources of magnetic field noise and potential systematic backgrounds in ARIADNE are associated with the magnetism of the source mass rotor itself. In this paper, we describe magnetometry measurements performed on the prototype tungsten source mass rotor to be used in the experiment. We study the frequency dependence of magnetic field as the rotor rotates within a magnetically shielded environment, and we show that the field is within a factor of 10 of the design requirements from the tungsten purity level and demagnetization process. We further show that the magnetic field profile from the rotor suggests the presence of a few discrete dipoles, likely due to impurities inside or on the rotor.

The magnetic background characterization methods and results we describe may be relevant for other existing and planned "fifth-force" experiments for novel exotic interactions, in particular, those requiring low background magnetic fields or nonmagnetic materials. Some examples include searches for new interactions between polarized electrons and unpolarized nucleons using comagnetometers $[10,11]$, precision torsion balances [12,13], other searches for monopole-dipole interactions with polarized nuclear spins and unpolarized nucleons using noble-gas comagnetometers [14,15], noble-gas-alkali-atom comagnetometers [10], and nuclear magnetic resonance [16], and searches for new short-range corrections to Newtonian gravity [17] and measurements of the Newton gravitational constant [18]. Tungsten is often a material of choice for tests of exotic mass-dependent interactions which couple to nucleons, given its high elastic modulus and high-nucleon density. Tungsten has been used in tests for novel gravitational interactions at submillimeter range [17], where magnetic field due to magnetic impurities or impurities of superconducting material deposits can be a significant concern. Tungsten alloy has also been used as a source mass in precision atom interferometric measurements of the Newton gravitational constant $G_{N}$ [18], where magnetic field backgrounds must be mitigated.

\section{EXPECTED MAGNETIC NOISE SOURCES}

The fundamental noise source which will limit ARIADNE's sensitivity is the quantum noise caused by the transverse spin projection in the ${ }^{3} \mathrm{He}$. For the target polarization level and nucleus density, this noise is expected to be around $3 \times 10^{-19} \mathrm{~T} / \sqrt{\mathrm{Hz}}\left(1000 \mathrm{~s} / T_{2}\right)^{1 / 2}$, where $T_{2}$ is the ${ }^{3} \mathrm{He}$ transverse relaxation time [7].

Besides the ${ }^{3} \mathrm{He}$ sample, the source mass produces numerous magnetic noises and backgrounds which must be lowered below the spin-projection noise so as to be spin-projection limited. We summarize the various noise and background requirements from the tungsten rotor [7], assuming a baseline shielding factor of $10^{8}$, an integration time of $10^{6} \mathrm{~s}$, and an axion sensitivity of $10^{-20} \mathrm{~T}$. A higher shielding factor or longer integration times can be targeted to reduce the magnetic effects of source mass and/or to probe deeper into the axion parameter space.

At the operating temperature of $170 \mathrm{~K}$ and at the modulation frequency $f_{\text {mod }}$ the contribution from thermal (Johnson) noise is expected to be around $10^{-12} \mathrm{~T} / \sqrt{\mathrm{Hz}}$ [7]. After shielding this will become $10^{-20} \mathrm{~T} / \sqrt{\mathrm{Hz}}$, or $10^{-23} \mathrm{~T}$ with integration.

Since the tungsten source mass is uncharged and being spun around its axis, it will acquire a magnetization due to the Barnett effect [19]. For rotation frequencies from 5 to $9 \mathrm{~Hz}$, we expect the field from this magnetization to be around $10^{-14} \mathrm{~T}$ [7], suppressed to $10^{-22} \mathrm{~T}$ with the shield. 


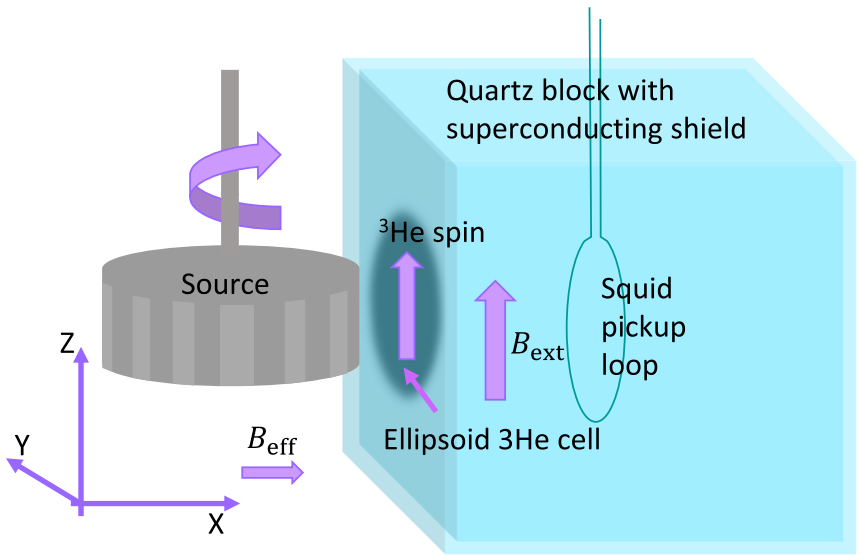

FIG. 1. Illustration of the ARIADNE concept; see Introduction for details.

Another source of magnetic backgrounds can be caused by magnetic impurities inside the tungsten source mass. To estimate this effect, we assume iron atoms trapped inside the source mass at a $1 \mathrm{ppm}$ purity level. The magnetic field from such impurities is calculated in Ref. [7] to be between $10^{-17}$ and $10^{-9} \mathrm{~T}$, which can be shielded to $10^{-25}-10^{-17} \mathrm{~T}$. The range comes from the distribution of the alignment of the Fe spins. This field at $1 \mathrm{ppm}$ impurity level is outside the specification if the spins are near-perfectly aligned. One of the aims of this study is to determine the typical magnetic impurity distributions in the prototype tungsten rotor. Effects of residual magnetism due to hysteresis from magnetizationdemagnetization cycles were studied in a previous publication and found to meet the requirements [20].

Finally, for a given background field, the intrinsic magnetic susceptibility of tungsten can lead to induced dipole moment. The rotation of the source mass can lead to a modulation of magnetic field from the induced moment at the Larmor frequency. For a background field of $10^{-10} \mathrm{~T}$, we expect a modulation with an amplitude of $10^{-14} \mathrm{~T}$ [7], which can be shielded to $10^{-22} \mathrm{~T}$ by the superconducting shield. This estimate can be used to assess how stringent the requirement on the background magnetic field in the experiment must be. For example, with these experimental parameters, for conducting an axion search at the $10^{-20}$ - T level, the background magnetic field thus should be maintained below the 10-nT level at the location of the sprocket. This can be implemented in the experiment by using a combination of magnetic shielding and magnetic shim coils. Furthermore, any effect due to the magnetic susceptibility of tungsten can also be distinguished in the final ARIADNE experiment by varying the value of the background magnetic field: Any axion-generated fictitious magnetic field will not depend on this background field, whereas the effect due to the magnetic susceptibility has a linear dependence.

\section{EXPERIMENTAL SETUP}

Our experimental setup consists of two optically pumped magnetometers (OPMs) placed in proximity to the source mass rotor, which is connected to a motor.

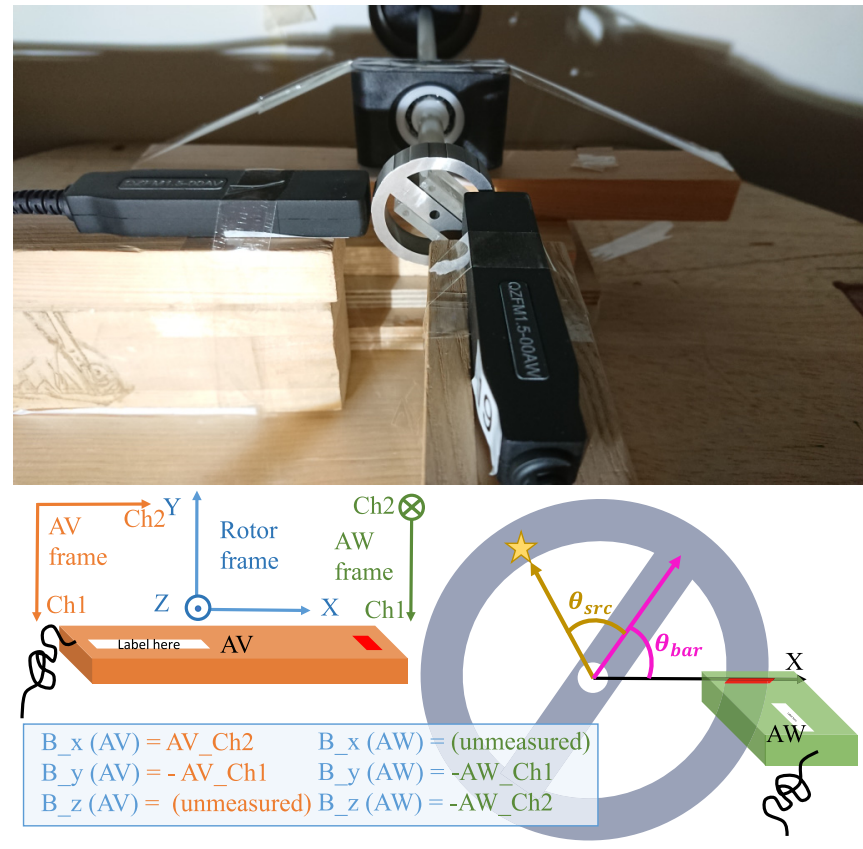

FIG. 2. Photograph (top) and schematic (bottom) of the setup. Two QuSpin OPMs, AV (orange) and AW (green), are placed in proximity to the rotating ARIADNE source mass. The source mass has $200-\mu \mathrm{m}$ cutouts along its circumference as can be seen in the photograph (not shown in the schematic). Each sensor is sensitive to magnetic field changes parallel to its long axis and its short axis within a small volume at its tip (shown in red). These orientations relative to the unified laboratory frame (blue) are also shown. $\theta_{\mathrm{bar}}$ denotes the angular location of the central bar on the source mass at the beginning of the measurement. $\theta_{\text {src }}$ denotes the location of an example impurity in reference to the bar. $\mathrm{Ch}$, channel.

The source mass has a height of $1 \mathrm{~cm}$, inner diameter of $3.02 \mathrm{~cm}$, and outer diameter of $3.8 \mathrm{~cm}$, divided into 22 equal sections. The section radii are alternately reduced by approximately $200 \mu \mathrm{m}$ in order to form a sprocket. Rotating the sprocket thus generates a time-varying potential at frequency $f_{\text {mod }}=11 f_{\text {rot }}$. The factor of 11 difference between $f_{\text {rot }}$ and $f_{\text {mod }}$ decouples mechanical vibration from the signal of interest. Tungsten that was more than $99.95 \%$ pure [21] was used to machine the source mass using wire electrical discharging machining [22]. To keep the axion signal at a constant level, the tolerance of the cutouts is chosen to be $\pm 10 \mathrm{~m}$. The source mass height is chosen to maximize overlap with the ${ }^{3} \mathrm{He}$ sample region. The smoothness of the top and bottom flat surfaces was achieved by finishing them with 220 and 440-grit abrasive sheets. The surface roughness was then measured using profilometry and found to be 1.4-2.1 m.

The rotor is demagnetized using a hard-drive eraser (Verity Systems, bulk degausser SV91m). It is then set up for rotation on an axle inside the Berlin magnetically shielded room-2 (BMSR-2) [23] driven by an outside motor. For this study the rotor was affixed to a glass-fiber-reinforced plastic shaft, held by a commercial glass or plastic bearing. In the ARIADNE experiment the sprocket will be driven by a $\mathrm{Ti}_{6} \mathrm{Al}_{4} \mathrm{~V}$ shaft and precision ceramic bearings to minimize tilt, wobble, and vibration. The two OPMs are placed next to the rotor. 
Field (pT) and positions of cut

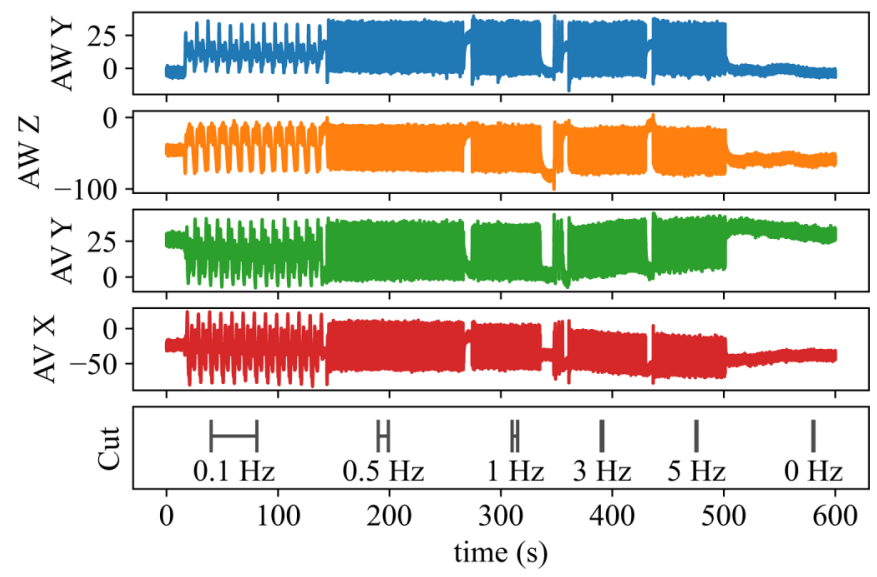

FIG. 3. Raw data from the magnetometers AW and AV, in the $y$ and $z$ directions for $\mathrm{AW}$, and in the $x$ and $y$ directions for $\mathrm{AV}$. The rotation is started at the slowest speed $(0.1 \mathrm{~Hz})$ at around $20 \mathrm{~s}$, and the speed is then increased in steps. In the bottom panel, we show the locations of the cut to extract a few cycles at each frequency for the time-domain analysis.

These QZFM Gen 1.5 OPMs manufactured by QuSpin have demonstrated measurements at the picotesla scale in a zero background field [24].

A photograph of the setup and a schematic are shown in Fig. 2. The sensor to the left of the rotor has been named AV, and the sensor in front of the rotor has been named AW. The OPM in the sensor is located at the red mark shown in the schematic.

The OPM used in this experiment measures the field at its location in two axes, depicted by the orange and green axes in the figure. In the laboratory frame, the sensor AW measures magnetic fields in the $y$ and $z$ directions, and the sensor AV measures magnetic fields in the $x$ and $y$ directions. The signals are sampled at $500 \mathrm{~Hz}$ and recorded at five rotation frequencies: $0.1,0.5,1,3$, and $5 \mathrm{~Hz}$. To establish measurement uncertainty, data were also obtained in the absence of rotation. The full data are shown in Fig. 3. The data are analyzed both in the time domain and in the frequency domain, each providing separate information.

\section{DATA ANALYSIS}

First we perform a spectral analysis to directly measure the magnetic field amplitude at the modulation frequency. This is done for both axes of both sensors and for all rotation frequencies. As an example, we show the fast Fourier transform (FFT) of the field measured by the sensor AV when the rotor is rotated at $5 \mathrm{~Hz}$ in Fig. 4. We summarize the field amplitude for all other frequencies as well as the AW sensor in Table I. We find that the amplitude of the magnetic field at the modulation frequency is below $2 \mathrm{pT}$ in all cases.

From the same spectral analysis, we can also obtain a rough upper limit on the magnetic field noise from the source mass. We can conservatively assume that the entire amplitude at $11 f_{\text {rot }}$ is caused by magnetic field noise and convert it to a spectral density. For the rotation speed of $5 \mathrm{~Hz}$, the measure-

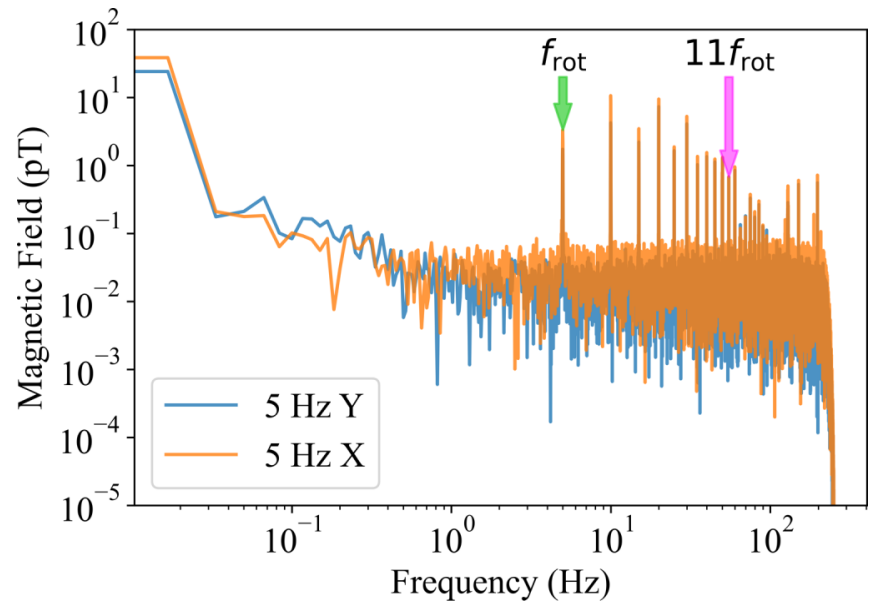

FIG. 4. Frequency spectrum of the magnetic field measured by the AV sensor in the $x$ and $y$ directions, with the rotor being rotated at $5 \mathrm{~Hz}$.

ment duration is $\sim 50 \mathrm{~s}$, giving a spectral density of roughly $4-5 \mathrm{pT} / \sqrt{\mathrm{Hz}}$ at the modulation frequency, $55 \mathrm{~Hz}$.

Although the frequency domain shows the field amplitude at the modulation frequency separately from other frequencies, it is not suitable to distinguish various noise or background sources. Specifically, it is unclear whether this field is explained by bulk noise mechanisms such as Johnson noise, by vibration noise, or by background field due to magnetic impurities.

In the time domain, we investigate whether the data can be significantly explained by a few isolated magnetic impurities inside the rotor. We do this by assuming that the impurities act as magnetic dipoles and fitting the locations and moments of such dipoles to the data. This information can then be used to estimate the contribution of impurities to the magnetic field at a distance closer to the rotor, as is the case for the intended axion measurement.

For the time-domain analysis, the data at each frequency are cut down and then averaged. Due to drifts in the rotation

TABLE I. Magnetic field amplitude at each sensor's location for both measurement axes. This amplitude has been obtained by converting the measurement to the frequency domain. For each pair of rows, the amplitude at the rotation frequency $f_{\text {rot }}$ is shown in the top row, and the amplitude at the modulation frequency $f_{\text {mod }}=11 f_{\text {rot }}$ is shown in the bottom row.

\begin{tabular}{lcccc}
\hline \hline Frequency (Hz) & AV $x(\mathrm{pT})$ & $\mathrm{AV} y(\mathrm{pT})$ & $\mathrm{AW} y(\mathrm{pT})$ & $\mathrm{AW} z(\mathrm{pT})$ \\
\hline 0.1 & 10.0 & 5.8 & 3.8 & 22.8 \\
1.1 & 1.7 & 1.3 & 0.6 & 0.5 \\
0.5 & 6.6 & 2.8 & 6.1 & 18.2 \\
5.5 & 1.1 & 0.9 & 0.6 & 0.5 \\
1 & 5.5 & 2.1 & 6.6 & 18.1 \\
11 & 0.8 & 0.7 & 0.7 & 0.5 \\
3 & 3.3 & 1.5 & 5.5 & 13.5 \\
33 & 0.6 & 0.6 & 0.6 & 0.5 \\
5 & 3.3 & 1.7 & 5.9 & 14.1 \\
55 & 0.7 & 0.7 & 0.6 & 0.5 \\
\hline \hline
\end{tabular}


Field as a function of rotation
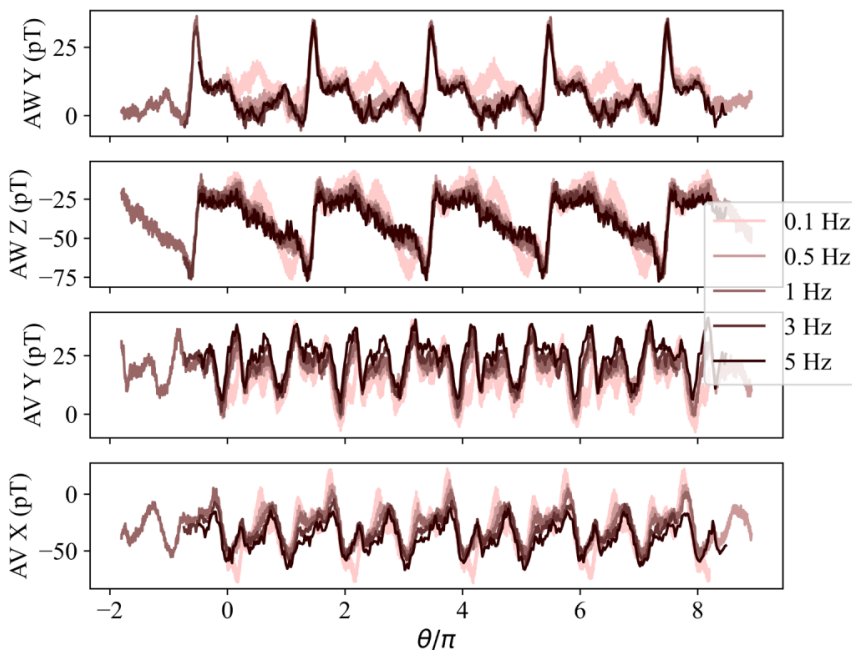

FIG. 5. A few cycles of data at all the rotation frequencies for both sensors and axes. Data for each frequency have been given a fixed shift in $\theta$ to make them line up.

speed, the averaging was done just over a few cycles. The locations of cuts are shown in the bottom panel of Fig. 3. After performing the cuts, the data from multiple frequencies can be compared by converting the time axis to a rotation angle. This is shown in Fig. 5, where we have added arbitrary time offsets to line up the measurements from different rotation frequencies. We see that all measurements but the one at $0.1 \mathrm{~Hz}$ are similar. We defer analysis of the cause of the deviation of the $0.1-\mathrm{Hz}$ measurement to future work, especially since this speed is too low to be relevant for the axion measurement.

For all other rotation frequencies, since the time-domain measurements line up, we can focus on analyzing any one of them. In this paper we focus on $5 \mathrm{~Hz}$ since it is the most relevant for the axion measurement [25]. The data at 5-Hz rotation frequency averaged over four rotation cycles are shown by the colored curves on the right side of Fig. 6 .

We now try to answer whether this measurement can be explained by a few discrete dipoles. For three dipoles, the problem is parametrized into $\kappa=23$ free parameters: the position and magnetic moments of each dipole $\left(6 \times N_{\text {dipoles }}\right)$, the dc offsets in the sensors $\left(N_{\text {sensors }} \times N_{\text {axes }}\right)$, and the initial location of the horizontal bar with respect to the $x$ axis in the laboratory frame $\left(\theta_{\text {bar }}\right)$.

Given the field from a magnetic dipole

$$
\mathbf{B}=\frac{\mu_{0}}{4 \pi}\left(3 \frac{\mathbf{m} \cdot \mathbf{r}}{r^{5}} \mathbf{r}-\frac{\mathbf{m}}{r^{3}}\right)
$$

for a source inside the rotor, rotation varies the position of the source $\mathbf{r}(t)$, giving a time-varying field $\mathbf{B}(t)$.

For each sensor and source, we define the problem in cylindrical coordinates as follows:

$$
\begin{aligned}
\mathbf{r}(t) & =\mathbf{R}_{\mathrm{OPM}}-\mathbf{R}_{\mathrm{src}}(t), \\
\mathbf{m} & =\left(m_{r}, m_{\theta}, m_{z}\right), \\
\mathbf{R}_{\mathrm{src}} & =\left(R_{\mathrm{src}}, \theta_{\mathrm{total}}(t), z_{\mathrm{src}}\right), \\
\theta_{\mathrm{total}}(t) & =\theta_{\mathrm{bar}}+\theta_{\mathrm{src}}+\theta_{\mathrm{rot}}(t), \\
\theta_{\mathrm{rot}}(t) & =\omega_{\mathrm{rot}}\left(t-t_{0}\right) .
\end{aligned}
$$

For a particular source moment and location as well as a known rotation speed, a vector magnetic field at the location of each OPM can be calculated using the above prescription. The total field at the location of $j$ th OPM will be the sum of fields from all dipoles:

$$
\mathbf{B}_{j}^{\text {model }}(t)=\sum_{i=1}^{N_{\text {dipoles }}} \mathbf{B}_{i j}(t) .
$$
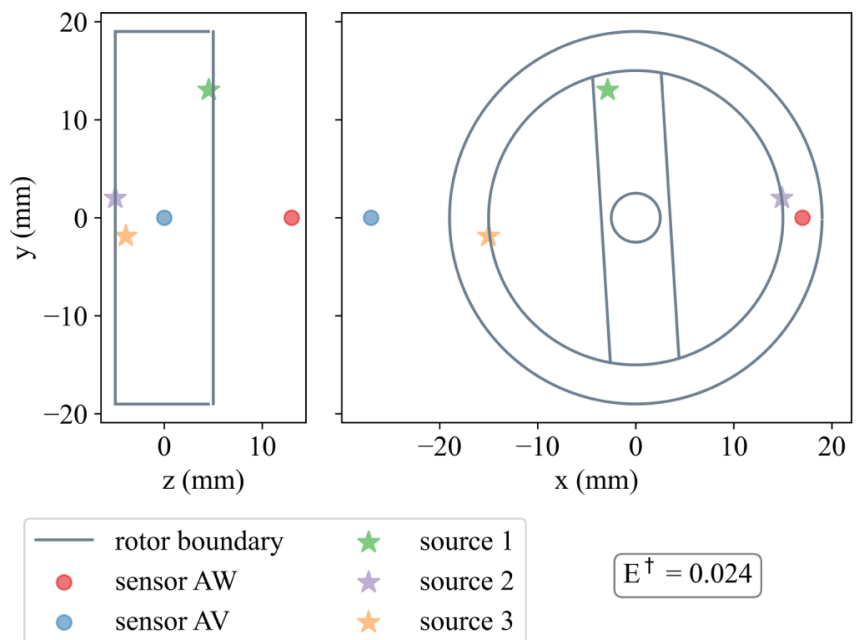

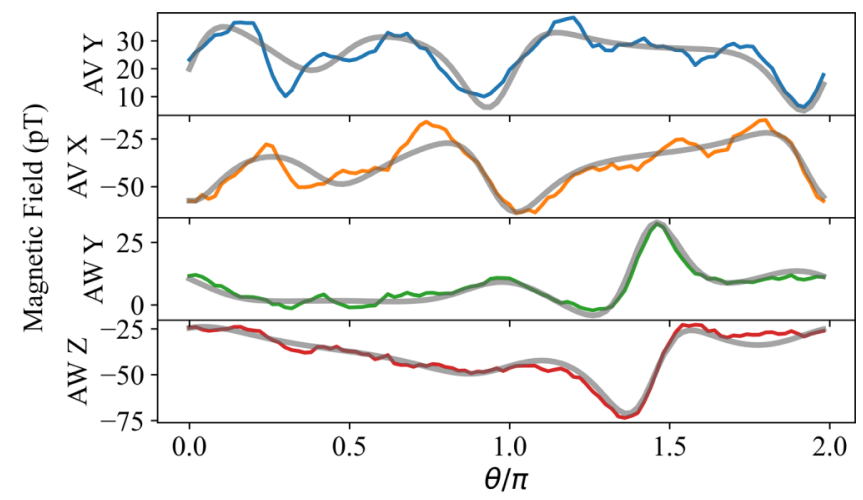

\begin{tabular}{|ccc|}
\hline Dipole \# & Position $^{\dagger}$ & Moment $^{\dagger}$ \\
1 & $\left(13 \mathrm{~mm}, 8.9^{\circ}, 4.5 \mathrm{~mm}\right)$ & $(14,-19,-21) \times 10^{-11} \mathrm{Am}^{2}$ \\
2 & $\left(15 \mathrm{~mm}, 270^{\circ},-5 \mathrm{~mm}\right)$ & $(13,-40,22) \times 10^{-11} \mathrm{Am}^{2}$ \\
3 & $\left(15 \mathrm{~mm}, 94^{\circ},-3.9 \mathrm{~mm}\right)$ & $(38,-30,-33) \times 10^{-11} \mathrm{Am}^{2}$ \\
\hline
\end{tabular}

FIG. 6. Fitting results. On the right, we show the recorded magnetic field data (see text for description of data processing) from each sensor with colored curves and the magnetic field produced by three hypothetical dipoles with gray curves. This is shown as a function of $\theta_{\text {rot }}$, the angle by which the rotor is rotated. On the left, we show a schematic of the problem: a front and side view of the rotor with each sensor's location indicated with a circle. The dipole locations and moments as defined in Eq. (5e) found by the fitting algorithm are listed at the bottom right. The locations are also indicated in the schematic as stars. †We report all fit parameters to two significant digits. It should, however, be noted that there is a measurement uncertainty of order $1 \mathrm{~mm}$ in the OPM locations which was not included in the fitting simulation. 
Then a normalized error function is defined:

$$
E=\frac{1}{N^{2}} \sum_{j, k, t}\left(\frac{B_{j k}^{\text {model }}(t)-B_{j k}^{\text {meas }}(t)}{\sigma_{j k}}\right)^{2},
$$

where $N$ is the total number of data points (396), $\sigma_{j k}$ is the measurement uncertainty for each sensor and axis (it is obtained from the $0-\mathrm{Hz}$ data and is in the range of $1 \mathrm{pT}$; further details can be found in Appendix), $j$ runs over the two OPMs ( $\mathrm{AV}$ and $\mathrm{AW}$ ), and $k$ runs over the two measurement axes of each OPM.

We then use the Nelder-Mead numerical algorithm [26] implemented by the PYTHON function scipy.optimize.minimize to find the parameters that minimize the above error function. We apply the constraint that the locations of the dipoles must lie in or on the rotor boundary. The result of the optimization is shown in Fig. 6. We show the fitted locations of the impurities in a schematic of the rotor, with their magnetic moments printed below. These dipole locations and moments minimize the error function $E$ to 0.02387 . The field calculated from these dipoles is shown (gray) in comparison to the measured field (color). The $\chi^{2} / \mathrm{DOF}$ for this fit (where DOF refers to degrees of freedom), obtained as $E N^{2} /(N-\kappa)$, is 10.0. The moments of the dipoles that minimize the error function have been bounded to be less than $10^{-9} \mathrm{~A} \mathrm{~m}^{2}$. The fit was also performed with a single dipole and two dipoles, but it failed to effectively capture the prominent features in the data. This can also be inferred because the three-dipole fit allots each of the dipole a magnetization of similar order of magnitude. We also tried to fit for a fourth dipole; however, the dipole moment that was returned was much smaller than the other three (roughly at the same place as deduced from the three-dipole fit), and the location was highly variable. This supports the hypothesis of three prominent dipoles.

After the moments and locations of the dipoles are obtained, we calculate the magnetic field due to these dipoles at the intended location of the ${ }^{3} \mathrm{He}$ sample in the ARIADNE experiment over a duration of about $10 \mathrm{~s}$. Subsequently, the contribution of the dipoles at the sample location can be extracted from the FFT of the calculated data at the modulation frequency $11 \times f_{\text {rot }}$. By this, we estimate that this contribution will be around $10 \mathrm{pT}$.

\section{CONCLUSION}

This work characterizes the magnetic noise and background caused by the source mass in the ARIADNE experiment. This field would have components at the same frequency as the fictitious axion field and would be completely indistinguishable from the signal; hence the only way forward is to reduce it below the expected axion interaction level.

We measured the magnetic field in proximity to the ARIADNE rotor after demagnetizing it. To understand the mechanism producing the field, we modeled the measurement as magnetic field created by three magnetic dipoles. We found good agreement between the measurement and the model, supporting the hypothesis that a significant portion of the measured field is created by trapped magnetic impurities at the rotor. We found that the contribution of the magnetic field amplitude at the location of the ${ }^{3} \mathrm{He}$ cells at the Larmor frequency

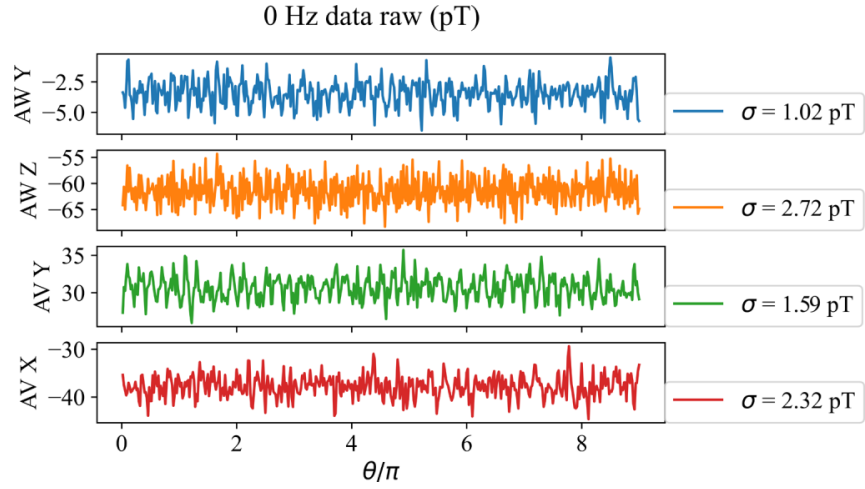

FIG. 7. Field measured in the absence of rotation, cut over a segment equal to the duration of the $5-\mathrm{Hz}$ data used for the time-domain analysis. The standard deviation for each trace is computed over this segment and displayed on the right. Time is converted to $\theta$ assuming a $5-\mathrm{Hz}$ rotation speed.

(which is 11 times the rotation frequency) is below $10 \mathrm{pT}$ and would require a shielding factor of $10^{9}$. A purer material, a better surface cleaning, or better demagnetization technique can be sought if this becomes a limiting background.

Going forward, with improved measurement precision on sensor location and orientation as well as lower vibration or wobble on the rotation assembly, the same data analysis method can be employed to obtain more precise information. In the future, the rotation speed can also be tracked by following the peaks over time in any one of the four channels. A separate channel that measures speed can also be deployed. An interferometric readout of the rotation speed is planned for the ARIADNE experiment [7]. This will allow for averaging over longer time as well as a more precise estimate of the contribution from Johnson noise vs coherent magnetic field background.

While the Nelder-Mead method is adequate to provide order-of-magnitude estimates of the dipole moments of trapped impurities and validates the hypothesis that a significant portion of the magnetic field noise is caused by a few discrete impurities, this method does have a number of limitations. For example, there are many degeneracies in the parameter space, making the method sensitive to the initial guess given to the optimizer. Multimodal solutions can be missed since the approach only provides one local minimum. In future, an approach based on the Markov-chain Monte Carlo method may provide results independent of the initial guess and better estimate the uncertainty in the magnitude and locations of magnetic impurities. Data with additional magnetometers at more spatial locations around the rotor could also help to improve the estimate of location and moments of the impurities.

The raw data as well as the code to perform all the analysis needed for this paper can be downloaded [27].

\section{ACKNOWLEDGMENTS}

We acknowledge support from the U.S. National Science Foundation, Grants No. NSF PHY-1509176, No. NSF PHY1510484, No. NSF PHY-1506508, No. NSF PHY-1806671, No. NSF PHY-1806395, and No. NSF PHY-1806757. A.B., 


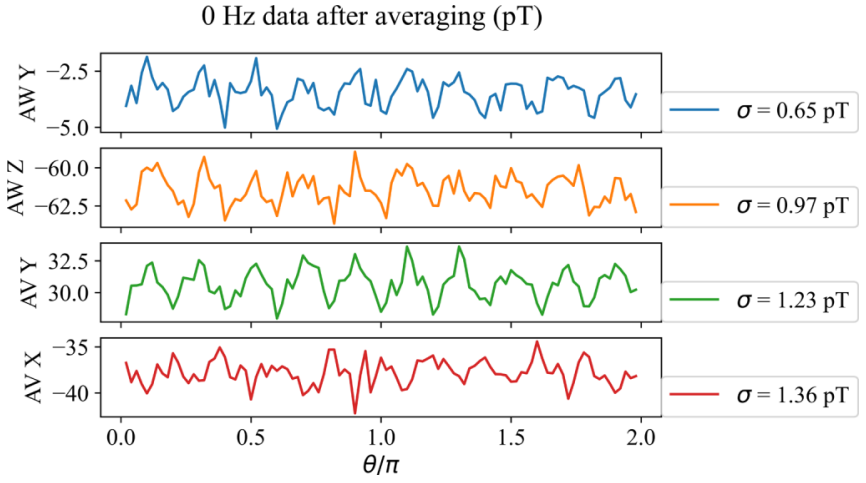

FIG. 8. Field in the absence of rotation, after averaging the data in Fig. 7. The standard deviations for each channel are lowered by the averaging and are shown on the right. Time is converted to $\theta$ assuming a 5-Hz rotation speed.

I.L., C.Y.L., J.C.L., A.R., J.S., E.S., and W.M.S. acknowledge support from the Indiana University Center for Spacetime Symmetries. Part of this work is supported by Institute for Basic Science under Grant No. IBS-R017-D1-2021-a00. We acknowledge the support of the Core Facility "Metrology of Ultra-Low Magnetic Fields" at Physikalisch-Technische Bundesanstalt, which receives funding from the Deutsche Forschungsgemeinschaft - DFG (Funding Codes No. DFG
KO 5321/3-1 and No. TR 408/11-1). We thank Gabriel Collin and Lutz Trahms for useful discussions.

A.B. and J.C.L. fabricated the source mass. A.S. and J.V. performed the measurements, N.A. analyzed the data and led this manuscript. All authors contributed to the final manuscript.

\section{APPENDIX: MEASUREMENT ERROR ESTIMATION}

In this Appendix we demonstrate how we obtained the measurement uncertainty $\sigma_{j k}$ used in Eq. (7). As shown in Fig. 3, data were also obtained without any rotation. A sample of these data was cut (location shown as the $0-\mathrm{Hz}$ label in Fig. 3). The length of the sample was chosen to be the same as the length of the 5-Hz sample. The $0-\mathrm{Hz}$ data after performing the cut are shown in Fig. 7, where the time axis for $0 \mathrm{~Hz}$ has been converted to $\theta$ for a $5-\mathrm{Hz}$ rotation speed.

The measurement uncertainty can be obtained by taking a standard deviation of the data in the absence of rotation. This standard deviation is also shown in Fig. 7. For the fitting of dipoles, the $5-\mathrm{Hz}$ data were averaged to reduce the errors. To estimate the error after averaging, $0-\mathrm{Hz}$ data were averaged in the same manner as $5-\mathrm{Hz}$ data, and a new standard deviation was calculated. The errors obtained after averaging, shown in Fig. 8, are then used in Eq. (7) to perform the optimization over dipole locations and dipole moments.
[1] R. D. Peccei and H. R. Quinn, CP Conservation in the Presence of Pseudoparticles, Phys. Rev. Lett. 38, 1440 (1977).

[2] S. Weinberg, A New Light Boson? Phys. Rev. Lett. 40, 223 (1978).

[3] F. Wilczek, Problem of Strong $p$ and $t$ Invariance in the Presence of Instantons, Phys. Rev. Lett. 40, 279 (1978).

[4] J. E. Moody and F. Wilczek, New macroscopic forces? Phys. Rev. D 30, 130 (1984).

[5] B. Graner, Y. Chen, E. G. Lindahl, and B. R. Heckel, Reduced Limit on the Permanent Electric Dipole Moment of ${ }^{199} \mathrm{Hg}$, Phys. Rev. Lett. 116, 161601 (2016).

[6] C. Abel, S. Afach, N. J. Ayres, C. A. Baker, G. Ban, G. Bison, K. Bodek, V. Bondar, M. Burghoff, E. Chanel, Z. Chowdhuri, P.-J. Chiu, B. Clement, C. B. Crawford, M. Daum, S. Emmenegger, L. Ferraris-Bouchez, M. Fertl, P. Flaux, B. Franke et al., Measurement of the Permanent Electric Dipole Moment of the Neutron, Phys. Rev. Lett. 124, 081803 (2020).

[7] A. Arvanitaki and A. A. Geraci, Resonantly Detecting AxionMediated Forces with Nuclear Magnetic Resonance, Phys. Rev. Lett. 113, 161801 (2014).

[8] A. Geraci et al. (ARIADNE Collaboration), Progress on the ARIADNE axion experiment, in Microwave Cavities and Detectors for Axion Research: Proceedings of the 2nd International Workshop, Springer Proceedings in Physics Vol. 211 (Springer, New York, 2018), pp. 151-161.

[9] H. Fosbinder-Elkins, Y. Kim, J. Dargert, M. Harkness, A. A. Geraci, E. Levenson-Falk, S. Mumford, A. Fang, A. Kapitulnik, A. N. Matlashov, D. Kim, Y. Shin, Y. K. Semertzidis, Y.-H. Lee, N. Aggarwal, C. Lohmeyer, A. Reid, J. Shortino, I. Lee, J. C. Long et al., A method for controlling the magnetic field near a superconducting boundary in the ARIADNE axion experiment, Quantum Sci. Technol. 7, 014002 (2022).

[10] J. Lee, A. Almasi, and M. Romalis, Improved Limits on Spin-Mass Interactions, Phys. Rev. Lett. 120, 161801 (2018).

[11] N. Crescini, C. Braggio, G. Carugno, P. Falferi, A. Ortolan, and G. Ruoso, Improved constraints on monopole-dipole interaction mediated by pseudoscalar bosons, Phys. Lett. B 773, 677 (2017).

[12] S. A. Hoedl, F. Fleischer, E. G. Adelberger, and B. R. Heckel, Improved Constraints on an Axion-Mediated Force, Phys. Rev. Lett. 106, 041801 (2011).

[13] W. A. Terrano, E. G. Adelberger, J. G. Lee, and B. R. Heckel, Short-Range, Spin-Dependent Interactions of Electrons: A Probe for Exotic Pseudo-Goldstone Bosons, Phys. Rev. Lett. 115, 201801 (2015).

[14] K. Tullney, F. Allmendinger, M. Burghoff, W. Heil, S. Karpuk, W. Kilian, S. Knappe-Grüneberg, W. Müller, U. Schmidt, A. Schnabel, F. Seifert, Y. Sobolev, and L. Trahms, Constraints on Spin-Dependent Short-Range Interaction between Nucleons, Phys. Rev. Lett. 111, 100801 (2013).

[15] M. Bulatowicz, R. Griffith, M. Larsen, J. Mirijanian, C. B. Fu, E. Smith, W. M. Snow, H. Yan, and T. G. Walker, Laboratory Search for a Long-Range $T$-Odd, P-Odd Interaction from Axionlike Particles Using Dual-Species Nuclear Magnetic Resonance with Polarized ${ }^{129}$ Xe and ${ }^{131}$ Xe Gas, Phys. Rev. Lett. 111, 102001 (2013).

[16] P.-H. Chu, A. Dennis, C. B. Fu, H. Gao, R. Khatiwada, G. Laskaris, K. Li, E. Smith, W. M. Snow, H. Yan, and W. Zheng, 
Laboratory search for spin-dependent short-range force from axionlike particles using optically polarized ${ }^{3} \mathrm{He}$ gas, Phys. Rev. D 87, 011105(R) (2013).

[17] D. M. Weld, J. Xia, B. Cabrera, and A. Kapitulnik, New apparatus for detecting micron-scale deviations from Newtonian gravity, Phys. Rev. D 77, 062006 (2008).

[18] G. Lamporesi, A. Bertoldi, L. Cacciapuoti, M. Prevedelli, and G. M. Tino, Determination of the Newtonian Gravitational Constant Using Atom Interferometry, Phys. Rev. Lett. 100, 050801 (2008).

[19] S. J. Barnett, Magnetization by rotation, Phys. Rev. 6, 239 (1915).

[20] C. Lohmeyer et al. (ARIADNE Collaboration), Source mass characterization in the ARIADNE axion experiment, in $\mathrm{Mi}$ crowave Cavities and Detectors for Axion Research: Proceedings of the 3rd International Workshop, Springer Proceedings in Physics Vol. 245 (Springer, New York, 2020), pp. 71-81.

[21] Source: Midwest Services, 540 Executive Drive, Willowbrook, Illinois 60527, USA.
[22] Wire electrical discharging machining performed by Stacy Machine and Tooling, Inc., 2685 Industrial Lane, Broomfield, Colorado 80020, USA.

[23] J. Nenonen, R. J. Iimoniemi, and T. Katila, Biomag 2000: Proceedings of the 12th International Conference on Biomagnetism, August 13-17, 2000 (Helsinki University of Technology, Espoo, Finland, 2001).

[24] J. Osborne, J. Orton, O. Alem, and V. Shah, Fully integrated standalone zero field optically pumped magnetometer for biomagnetism, in Steep Dispersion Engineering and Opto-Atomic Precision Metrology XI, Proceedings of SPIE Vol. 10548, edited by S. M. Shahriar and J. Scheuer (SPIE, Bellingham, WA, 2018), pp. 89-95.

[25] The analysis for all other frequencies can be found in the data and code released with this paper.

[26] F. Gao and L. Han, Implementing the Nelder-Mead simplex algorithm with adaptive parameters, Comput. Optim. Appl. 51, 259 (2012).

[27] N. Aggarwal, nancy-aggarwal/Characterization-of-ARIADNEsource-mass-rotor_Github: First release, https://doi.org/10. 5281/zenodo.5520151, 2021. 